\title{
Maritime UAVs' Swarm Intelligent Robot Modelling and Simulation using Accurate SLAM method and Rao- Blackwellized Particle Filters
}

\author{
Hadi Karimian and Amir Anvar \\ School of Mechanical Engineering \\ The University of Adelaide, \\ Adelaide, SA 5005 \\ AUSTRALIA \\ Emails: Hadi.Karimian@student.adelaide.edu.au ; Amir.anvar@adelaide.edu.au
}

\begin{abstract}
The objective of this research-study is to explore the performance of Rao-Blackwellized Particle Filters for accurate, simultaneous localization and mapping (SLAM), with Swarm Intelligent network of UAV Robots in oceanic environment.
\end{abstract}

SLAM is a method for Mobile-robots such as Maritime-UAVs Robots which could be valuable and effective to build-up a map on an unknown oceanic-air environment. In this aim, a variety of methods can be implemented and suggested by scientists. The Kalman Filter, Extended Kalman Filter, and Particle Filter are well known and popular algorithm techniques. Each of the named methods, are able to investigate on SLAM problem but may have some drawbacks, with working under some assumptions, that are not always true. As an example Extended Kalman filter estimates covariance matrix, that tends to underestimate the true covariance matrix, therefore it risks becoming inconsistent in the statistical sense.

Therefore, to get a good and accurate result, it is necessary to combine several methods together. That is included with Rao-Blackwellized method, with ability to construct Particle Filter and Extended Kalman Filter for SLAM scenarios. The Particle Filter technique is responsible for estimating Robot's pose as the Extended-Kalman-Filter estimates the landmarks.

Generally in marine applications UAVs are used for search and rescue mission(s), damage assessment, Maritime air environmental study and Maritime security reconnaissance which in most cases they need to cover an immense area of ocean(s). Therefore, time-wise using a single UAV is inefficient and there could be a risk of mission failure as a result of UAV's energy limitations as well as diagnostic problems. So, having a team of UAVs which each one can produce and follow its own map with cooperation with the others to satisfy mission goals would be much more efficient. The paper discusses the process of implementing, modelling and Matlab-simulation of the above techniques and shows the concept benefits in further studies of ocean-air Robotics application scenarios.

Keywords: Rao-Blackwellised Particle Filter (RBPF), Swarm, Intelligent, FastSLAM, Simultaneous Localization and Mapping (SLAM), Unmanned Aerial Vehicle (UAV), Ocean, Maritime, and Robot 
Hadi Karimian and Amir Anvar, Maritime UAVs' Swarm Intelligent Robot Modelling and Simulation using Accurate SLAM method and Rao-Blackwellized Particle Filters

\section{INTRODUCTION}

Simultaneous Localisation and Mapping (SLAM), for mobile-robots, could be used to address the problem of creating an accurate map of the robots' pose and the environment of robots' activity. This issue plays an important role for the robots in order to conduct a task successfully. In this aim, a variety of methods that are suggested by scientists, such as The Kalman Filter (KF), Extended Kalman Filter (EKF), and Particle Filter, were investigated. Although the previously mentioned methods are used widely, in most cases they are not capable of providing the robots with an accurate map. Therefore, some more accurate algorithms have emerged that are commonly composed of the methods that were mentioned above, such as RaoBlackwellized Particle Filter (RBPF). The RBPF is the main concentration of this research study for an accurate SLAM that consists of Particle Filter and EKF. In this method Particle Filter estimates the pose of the robot, while the EKF estimates the object's location within the environment.

Several research studies have been conducted towards the RBPF to improve its capability and accuracy. D. Hahnel et al [1] proposed an algorithm to introduce a reduction in the number of particles in the RBPF by using Scan Matching. Scan matching is used to transform a sequence of laser scans into odometry measurements that minimizes the odometric errors during mapping. G. Crisetti et al [2] proposed an algorithm for the RBPF based on grid SLAM with improved proposal distribution and adaptive re-sampling. FastSLAM 2.0 uses Rao-Blackwellized particle filter that utilises Gaussian approximation of the improved proposal for a feature (Landmark) based SLAM. Authors in [2] propose an efficient algorithm for Resampling, since it has a major influence on the performance of the particle filter.

Swarm intelligence in robotics research is the concept of multi-robot systems (system-of-systems), which focus on physical incorporation, and realistic interactions among the robots themselves, the other robots and the environment. Beni [3] used the word swarm for the first time to describe a special type of cellular robotic system in 1989. Swarm intelligence algorithms in robotics are mostly inspired by nature. Some well-known algorithms include: Ant Colony Optimization (ACO), Particle Swarm Optimization (PSO), Genetic Algorithms (GA) and Artificial Bee Colony (ABC).

The methods mentioned above are often computationally expensive and exhibit some deficiencies in some cases which may take a long time to overcome or may even remain unfixable. Therefore, some new methods were developed to compensate their shortage or inabilities by combining these basic algorithms together, such as Hybrid Ant Colony Optimization (ACO)/Particle Swarm Optimization (PSO). This case study is focused on Hybrid (ACO) / (PSO) control algorithm for distributed Swarm Intelligence.

This paper discusses the process of implementing, modelling and Matlab-simulation of the RBPF and Hybrid (ACO) / (PSO) in order to create a Unified Network for Swarm UAVs and shows its benefits in further studies of ocean-air robotics application scenarios.

\section{THE RBPF FOR SLAM:}

This research study investigates the SLAM solution based on the RBPF. The RBPF uses a particle filter to sample over robot paths [4]. Each particle carries $N$ low-dimensional EKF where $N$ is the number of landmarks (or object of interest within search enclosure) in the environment (one for each of the $N$ landmarks). This representation requires $O(N \times M)$ memory, where $M$ is the number of particles in the particle filter formula. Updating this filter requires $O(M \log N)$ time, with or without knowledge of the data association. However, the number of particles $(M)$ which is required for convergence is unknown. As, it has been pointed out in [35] [46], the SLAM posterior for the RBPF can be factored as:

$$
P\left(s^{t}, \Theta \mid y^{t}, u^{t}\right)=p\left(s^{t} \mid y^{t}, u^{t}\right) \prod_{n=1}^{F} p\left(\Theta_{n} \mid s^{t}, y^{t}\right)
$$

Where:

$\Theta=$ landmark position,

$s^{t}=$ sampled robot pose (particles)at $\mathrm{t}$,

$\mathrm{F}=$ separate estimates of feature locations,

$u^{t}=$ control signal at time $\mathrm{t}$,

$\mathrm{y}^{\mathrm{t}}=$ observation at time $\mathrm{t}$. 
In the RBPF, the posterior over the robot's path is estimated using a particle filter, since it only considers a subspace of the possible space. The remaining $F$ posterior of the feature's locations (which are conditional on the sampled robot pose $s_{t}$ ) are calculated using the EKF. Since each EKF estimates a single landmark position $\left(\Theta_{\mathrm{x}}^{\mathrm{i}}, \Theta_{\mathrm{y}}^{\mathrm{i}}\right)$, it is low-dimensional [7].

The robust RBPF has three main components. Those components are an accurate motion model, observation model and a reliable proposal distribution. The proposal distribution for simulation in this project, involved with the sampling distribution, is defined as follows:

$$
s_{t}^{[M]} \sim p\left(s^{t} \mid s^{[M]}{ }_{t-1}, u^{t}\right)
$$

Equation (2) samples the pose $s_{t}$ in accordance with $M^{\text {th }}$ particle, by drawing a sample according to the model of the motion.

In this study, to simulate the real-time condition(s), two noises, $W_{U}$ and $R_{Z}$, are artificially added to the equations of motion and observation of the UAV. Where, $W_{U}$ is a Gaussian control (or process) noise with a zero-mean and $R_{Z}$ is a Gaussian observation noise with a zero-mean. To simplify the simulation and creation of the virtual map, a series of assumptions have been made in terms of the UAV's equations of motion and observation, which are as follows:

- It's assumed that the UAV moves in a two-dimensional Cartesian reference frame (earth). Therefore, the space state vector, $X=\left[x_{t}, y_{t}, \phi_{t}\right]^{T}$, represents the simplified position and orientation of a UAV. Based on this assumption the position of the objects of interest (or landmarks) could be defined by vector $L_{i}=\left[x_{i}, y_{i}\right]^{T}$.

- The assumptions of having a constant velocity $(V \mathrm{~m} / \mathrm{s})$ and only having control over the orientation, of the UAV have been made.

Based on the above assumptions, the equation of motion of the UAV in two-dimensional Cartesian frame could be written as:

$$
\left[\begin{array}{l}
x_{t} \\
y_{t} \\
\phi_{t}
\end{array}\right]=\left[\begin{array}{c}
x_{t-1}+\Delta t \cdot V \cdot \cos \left(\phi_{t-1}+U_{t}\right) \\
y_{t-1}+\Delta t \cdot V \cdot \sin \left(\phi_{t-1}+U_{t}\right) \\
\phi_{t-1}+U_{t}
\end{array}\right]+W_{u}
$$

Where:

$x_{t-1}$ is the position of UAV at time $\mathrm{t}-$

1 , respect to the $x$ - axis,

$y_{t-1}$ is the position of UAV at time $\mathrm{t}-$

1 , respect to the $\mathrm{y}-$ axis,

$\phi_{t-1}$ is the orientation of UAV at time,

Regarding the assumption of moving in a two-dimensional Cartesian reference frame, the observation vector of the UAV could be defined by $Z_{t}=\left[r_{t}, \theta_{t}\right]$. Where, $r_{t}$ is the observation range in meters and $\theta_{t}$ is the observation bearing in radian. Therefore, the equation of observation for the UAV could be defined as:

$$
\left[\begin{array}{l}
r_{t} \\
\theta_{t}
\end{array}\right]=\left[\begin{array}{c}
\sqrt{\left(x_{i}-x_{t}\right)+\left(y_{i}-y_{t}\right)} \\
\operatorname{atan}\left(y_{i}-y_{t} / x_{i}-x_{t}\right)-\phi_{t}
\end{array}\right]+R_{Z}
$$

It needs to be considered that in reality the equations of motion and observation are more complicated, and that is due to UAV's movement in a three dimensional Cartesian frame. In addition, mostly, in reality the control and the observation noises are not Gaussian zero-means. The filtering steps, which were used in the RBPF simulation, have been summarized as follows:

Prediction: This step uses equations (3) and (4) to create the configuration of each particle.

Since each particle represents a small portion of the map, therefore, there is multi hypothesis. The final configuration of particles can be shown in the Table1: 
Hadi Karimian and Amir Anvar, Maritime UAVs' Swarm Intelligent Robot Modelling and Simulation using Accurate SLAM method and Rao-Blackwellized Particle Filters

Table 1. Configuration of Samples (or particles)

\begin{tabular}{|l|c|l|}
\hline Particle & Robot's pose & $\begin{array}{l}\text { Mean and covariance } \\
\text { of each landmark }\end{array}$ \\
\hline 1 & $s_{x}^{1}, s_{y}^{1}, s_{\theta}^{1}$ & $\mu_{1, t}^{[1]}, \Sigma_{1, t}^{[1]}, \ldots, \mu_{N 1, t}^{[1]}, \Sigma_{N 1, t}^{[1]}$ \\
\hline 2 & $s_{x}^{2}, s_{y}^{2}, s_{\theta}^{2}$ & $\mu_{1, t}^{[2]}, \Sigma_{1, t}^{[2]}, \ldots, \mu_{N 2, t}^{[2]}, \Sigma_{N 2, t}^{[2]}$ \\
\hline $\mathrm{M}$ & $s_{x}^{M}, s_{y}^{M}, s_{\theta}^{M}$ & $\mu_{M, t}^{[M]}, \Sigma_{M, t}^{[M]}, ., \mu_{N_{M}, t}^{[M]}, \Sigma_{N_{M}}^{[M]}$ \\
\hline
\end{tabular}

Figure (1) illustrates a graphical demonstration of Table (1). It shows each particle may contain different numbers of landmarks.

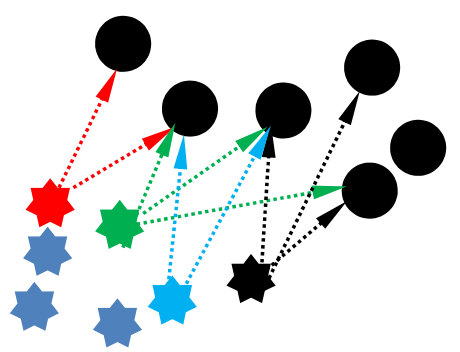

Figure 1. Coloured Stars are particles and black circles are landmarks (or objects of interest). Dotted arrows are showing the observation of each particle of landmarks.

Jacobian Observation: Using the Jacobian definition, the Jacobian of observation $(\mathrm{H})$ could be derived from (4):

$$
\mathrm{H}=\nabla\left[\begin{array}{l}
\mathrm{r}_{\mathrm{t}} \\
\theta_{\mathrm{t}}
\end{array}\right]=\left[\begin{array}{cc}
\frac{\mu_{\mathrm{x}, \mathrm{t}}^{\mathrm{M}}-\mathrm{s}_{\mathrm{x}, \mathrm{t}}^{\mathrm{M}}}{\mathrm{r}_{\mathrm{t}}} & \frac{\mu_{\mathrm{y}, \mathrm{t}}^{\mathrm{M}}-\mathrm{s}_{\mathrm{y}, \mathrm{t}}^{\mathrm{M}}}{\mathrm{r}_{\mathrm{t}}} \\
-\frac{\mu_{\mathrm{y}, \mathrm{t}}^{\mathrm{M}}-\mathrm{s}_{\mathrm{y}, \mathrm{t}}^{\mathrm{M}}}{\mathrm{r}_{\mathrm{t}}{ }^{2}} & \frac{\mu_{\mathrm{x}, \mathrm{t}}^{\mathrm{M}}-s_{\mathrm{x}, \mathrm{t}}^{\mathrm{M}}}{\mathrm{r}_{\mathrm{t}}{ }^{2}}
\end{array}\right]
$$

Where:

(5) $\mu_{x, t}^{M}$ and $\mu_{y, t}^{M}$ are the $\mathrm{x}$ and $\mathrm{y}$ mean- coordinates of Landmarks.

$r_{t}$ is the first term of (4)

Association: The Known Data Association in this research study has been done based on the common method of the maximum likelihood (ML). It is defined in the following equation:

$$
M L=\left|2 \pi Q_{n}\right|^{\frac{-1}{2}} \exp \left\{-0.5\left(z_{t}-\widehat{z_{N}}\right)^{T} Q_{n}^{-1}\left(z_{t}-\widehat{z_{N}}\right)\right\}
$$

Where:

$\widehat{Z_{N}}$ is the measurement estimation which is calculated using equation (4),
$Q_{n}$ is the measurement covariance that could be calculated by:

$$
Q_{n}=H^{T} \Sigma_{N, t-1}^{[M]} H+R_{Z}
$$

New Landmark: The detection of a new landmark has been implemented by defining a threshold value regarding to the data association. If the ML of the observed landmark drops down below a threshold value, that landmark is considered to be new landmark. Therefore, its mean and covariance would be inversed to initialise the mean and covariance of the landmark in the EKF. [4]

Update Landmark: This step is accomplished using the equations of the EKF for updating. [8] [9]

For the RBF simulation, the steps defined above need to be carried out sequentially in a loop and the output estimation of the robot's path and map needs to be stored as $t-1$ data ( $\mathrm{t}$ is a counter that can be measured in seconds or the number of iterations). This data set will be retrieved in the next iteration or time step to extract required information for new estimations of pose and map.

\section{HYBRID ACO/PSO:}

The main aim of this hybrid method is to achieve an optimal Swarm behaviour for a large number of robots. Each robot adjusts its movement behaviour based on the target's utility function and shared information through local communication. The Target utility function has been defined as the fitness value of moving to different areas based on a number of parameters such as Pheromones, agent intensity, pheromone evaporation function, and potential weight for each pheromone [10]. These new meta-heuristics draw on the strengths of both systems: ACO's autocatalytic mechanism through environment and PSO's cognitive capabilities through the interplay among agents. Payton et al. [11] proposed Virtual Pheromones in robotics. Their model got its inspiration from chemical insects, such as ants. (Ants use chemical pheromones to communicate). Meng et al. [12] implemented a network for distributed swarm robots by Hybrid ACO/PSO control algorithm. In their work, the virtual pheromones are like messages that pass coordination schemes for the swarm robots. The mathematical model of Hybrid ACO/PSO, which is proposed by the authors in [12], for ACO subsection, uses the classical equations and definitions of agent intensity, pheromone's evaporation and the target's utility for each agent in swarm. These equations and definitions are as follows: 
Hadi Karimian and Amir Anvar, Maritime UAVs' Swarm Intelligent Robot Modelling and Simulation using Accurate SLAM method and Rao-Blackwellized Particle Filters

$$
\tau_{i j}^{k}(t+1)=\rho \cdot\left(\tau_{i j}^{k}(t)+T_{i j}^{k}\right)-(1-\rho) e \tau_{i j}^{k}(t)(7) \quad \mu_{i j}^{k}(t)=\left(K_{1} \omega_{i j}^{k}(t)-K_{2} \tau_{i j}^{k}(t)\right) / R
$$

Where:

Where:

$\rho$ is the enhancement factor of pheromone intensity, $T_{i j}^{k}$ is the pheromone interaction intensity,

$$
T_{i j}=\left\{\begin{array}{lr}
\alpha, & \text { if source pheromone } \\
\beta, & \text { otherwise }
\end{array}\right.
$$

$0 \leq \beta<\alpha \leq 1$

$\omega_{i j}^{k}(t)$ and $\tau_{i j}^{k}(t)$ are target weight and agent intensity,

$R$ is the local target redundancy,

$K 1$ and $K 2$ are constant factors that used to adjust the weights of target weight and agent intensity parameters,

\section{to each Virtual Pheromone,}

e represents the elimination factor,

In addition, for PSO subsection of Hybrid ACO/PSO, they used the following equations and definitions to calculate the visibility, velocity and the position of each agent in swarm at each iteration or time $t$ respective to each pheromone:

$$
\eta_{i j}^{k}(t)=r^{k} / d_{i j}^{k}(t)
$$

Where:

$$
k \text {, }
$$

$r^{k}$ represents the local detection range of the UAV

$$
\begin{aligned}
& d_{i j}^{k}(t) \text { represents the distance between the UAVS } \\
& \text { and target }
\end{aligned}
$$

The velocity of each agent in $\mathrm{t}+1$ second is calculated as follows:

$$
v_{i j}^{k}(t+1)=\psi_{e} \operatorname{rand}_{e} v_{i j}^{k}(t)+\psi_{c} \operatorname{rand}_{c}\left(p_{c}-x_{i j}^{k}(t)\right)+\psi_{s} \operatorname{rand}_{s}\left(p_{s}-x_{i j}^{k}(t)\right)
$$

Where :

$v_{i j}^{k}(t)$ is the velocity of an UAV, $\psi e, \psi c$, and $\psi s$ : Represent the propensity constraint factors for explorer, cognitive, and social behaviours. Respectively,

The position adopted by UAV $k$ at time $t+1$ is updated by:

$x_{i j}^{k}(t):$ Represents the position of UAV $k$ at a time $t$,

$p_{s}=\max \left(\eta_{i j}^{k}(t)\right):$ Represents the global best from the neighbours, $p_{c}=\max \left(\eta_{i j}^{k}(t)\right):$ Represents the local cognitive best

$$
x_{i j}^{k}(t+1)=x_{i j}^{k}(t)+v_{i j}^{k}(t+1)
$$

\section{SIMULATION PSEUDO CODE OF UNIFIED NETWORK:}

In the algorithm that has been proposed and simulated in this study, each member of the Swarm UAVs can sense other members in the neighbourhood and is permitted to communicate just by the control-centre. Therefore, the designed network is a branch of a centralized communication and control system by noncommunicative members. The advantage of this network is introducing a reduction in number of mounted sensors in UAVs that makes them lighter and reduces the energy consumption.

As Swarm UAVs flying over the search enclosure, each member of Swarm UAVs will create and estimate its map independently by using the RBPF. Data from each member will be sent to the control-centre. Received data will be analysed in the control-centre using Hybrid ACO/PSO algorithm. Then, the results will appear on the human operator's map. Finally, if any specific action is required, based on the mission's goal, the human operator will send an order to each desired member. It is notable that in Hybrid ACO/PSO each landmark (or object of interest), plays the role of a pheromone. This study takes the advantage of virtual pheromones, proposed by the authors in [11], and modified version of Hybrid ACO/PSO algorithm, which is proposed by the authors in [12]. There is no random search in the modified algorithm of Hybrid ACO/PSO.

The designed network can be summarised in a pseudo code as follows:

At time $=0$

Initialise Pheromones matrices for each UAV

Set propensity constraint factors to zero

$\left(\psi_{e}, \psi_{c}, \psi_{s}\right)$

Initialise Swarm UAVs speed (10)

Initialise Swarm UAVs position (11)

$t=0$; flag $=0$;

\#while loop $t=0$; flag $=0$;

\#while loop

While flag $=0$

$t=t+1$;

For 1: $k$

Do the RBPF (k) for SLAM defined in section 2

End for

At time each instant of time 
Hadi Karimian and Amir Anvar, Maritime UAVs' Swarm Intelligent Robot Modelling and Simulation using Accurate SLAM method and Rao-Blackwellized Particle Filters

While flag $==0$

of Swarm UAVs add it to pheromone metric by

Swarm member's Index

At time each instant of time

If any object (or landmark) detected by any member of Swarm UAVs add it to pheromone metric by

Swarm member's Index

Check all pheromone data that have been obtained from Swarm UAVS

If any detected-object (or landmark) is shared by two or more members then:

Calculate $\tau_{a^{k}}(t)$ (Number of UAVS who has seen the landmark)

Calculate related pheromone evaporation from

(7) by setting $\alpha=1$ and $\rho=0<$ rand $<1$

Calculate related weight for each pheromone

based on its distance from each member who detected the landmark $\left(\omega_{a^{k}}\right)$

Calculate the utility of each detected-Landmark by

(8) $\left(\mu_{i j}^{k}\right)$

Calculate the local visibility of an object by (9) $\left(\eta_{i j}^{k}\right)$

$\operatorname{Set}\left(\psi_{e}, \psi_{c}, \psi_{s}\right)$ to predefined values and Update the speed of each agent by (10)

Update the position of the agents by (11)

Else, if any detected-object (or landmark) is not
If any object (or landmark) detected by any member shared by two or more members then:

Set $T_{a^{k}}(t)=1$ for the member saw the object

Calculate related pheromone evaporation from

the equation (7) by setting $\alpha=.5$ and $\rho=0<$

Rand $<1$

Calculate related weight for each pheromone based on its distance from member who detected the landmark $\left(\omega_{a^{k}}\right)$

Calculate the utility of each detected-Landmark by (8) $\left(\mu_{i j}^{k}\right)$

Calculate the local visibility of an object by (9) $\left(\eta_{i j}^{k}\right)$

Set $\psi_{e}=0, \psi_{c}=1, \psi_{s}=0$ and Update the speed of related agent by equation (10)

Update the position of related agent by equation

End else if

End if

End if

Show results on display

If the mission's requirement satisfied Flag=1;

End if

End of while

\section{SIMULATION RESULTS:}

The following figure 2, demonstrates a sample of simulation for the RBPF, defined in section two with 120 particles, in a virtual environment containing 8-landmarks. The same test has been carried out for different numbers of particles with same number of landmarks. Results are tabulated in table 2:

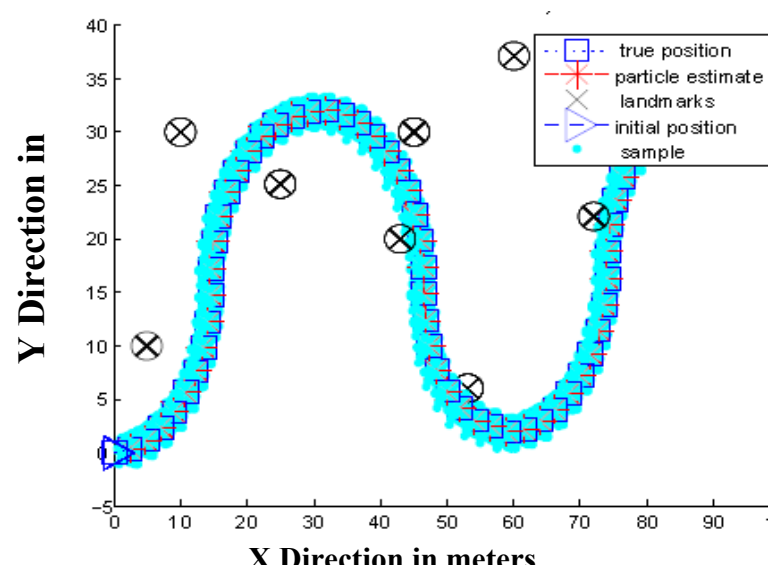

Figure 2. Simulduon resuı will $1 \angle \mathrm{U}$ particles. the black circles with crosses in the map show detected landmarks or object of interest in search enclosure.

Table 2. results of the RBPF simulation regarding to the number of particles and absolute related error:

\begin{tabular}{|c|c|c|c|c|}
\hline $\begin{array}{c}\text { Number } \\
\text { of } \\
\text { particles }\end{array}$ & $\begin{array}{c}\text { Error } \\
\text { in X } \\
(\mathrm{m})\end{array}$ & $\begin{array}{c}\text { Error } \\
\text { in Y } \\
(\mathrm{m})\end{array}$ & $\begin{array}{c}\text { Error in } \\
\text { angle } \\
\text { (degree) }\end{array}$ & $\begin{array}{c}\text { Number of } \\
\text { detected } \\
\text { objects }\end{array}$ \\
\hline 40 & $20 \%$ & $22 \%$ & $14 \%$ & 6 \\
\hline 120 & $\sim 2 \%$ & $1.5 \%$ & $2 \%$ & 8 \\
\hline 300 & $0.4 \%$ & $\sim 0.5 \%$ & $0.3 \%$ & 8 \\
\hline
\end{tabular}

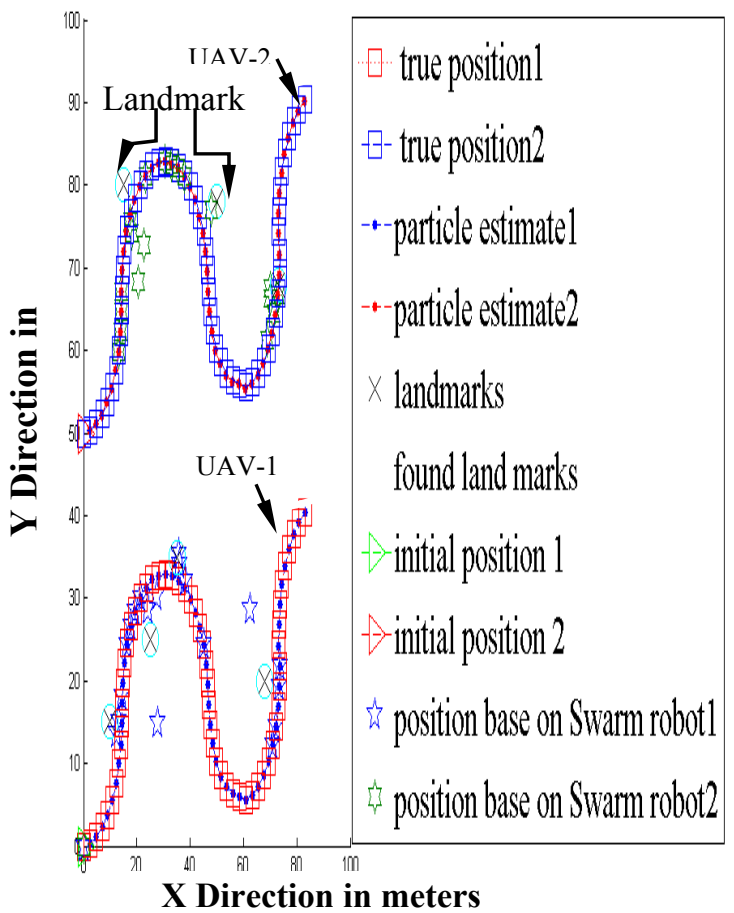

Figure 3. The result of search the desired enclosure by Swarm UAV 
To test the performance of the designed network, defined in section four, a virtual environment with 7 scattered landmarks is created. The attempt of covering the whole searching enclosure just by one UAV has failed; therefore the simulation has been repeated for two UAVs in the same environment. Figure 3, illustrates the result of the second test by two UAVs and shows the searching enclosure which is completely covered by Swarm UAVs. By close inspection, some blue and green stars as appears on searching area, those stars are showing the next best position for each member of Swarm with respect to the landmarks, calculated by Hybrid ACO/PSO analysis. Whenever a landmark is detected, immediately a blue star for UAV-1 and a Green Star for UAV-2 would appear on the display. Those points are coordinates that are chosen by Hybrid $\mathrm{ACO} / \mathrm{PSO}$ algorithm based on cognitive, exploration and the social behaviour of UAV-1 and UAV-2. When a landmark is detected by UAV-1 a blue star appears close to detected landmark to highlight the next best position for UAV-1, at the same time a green star becomes visible on the path of UAV-2 which is far away from the detected landmark by UAV-1. It shows that the best action for UAV-2 is to keep on searching for other landmarks.

\section{CONCLUSION}

This paper represents a theoretical model of designing a unified network for swarm UAVs. The research has studied the functionality of the RBPF method for solving the SLAM problem, as well as the utility of the Hybrid ACO/PSO algorithm for swarm intelligence in robotics. From the tabulated results in table 2, for the RBPF simulation, it can be concluded that a higher number of particles in the RBPF results in a better poseestimation and landmarks detection for SLAM. However, it is a trade off between the number of particles and the amount of calculation that needs to be carried out by robots. In addition, the performance of designed network for swarm UAVs is proved by obtained results from matlab-simulation. Therefore, it could be concluded that, the advised algorithm is an efficient method to implement in real time. Furthermore, a sucessful simulation was carried out to replicate the transition between Swarm UAVs and the control-centre. In addition, it is assumed that the whole performance is affected by Gaussian zero-mean noises.

\section{REFERENCES}

[1] D. Hahnel, W. Burgard, D. Fox, and S. Thrun. ( 2003)."An efficient, fastslam algorithm for generating maps of large-scale cyclic environments from raw laser range measurements".

[2] G. Crisetti, C. Stachniss and W. Burgard.( 2007)."Improved techniques for grid mapping with Raoblackwellized particle filters.” IEEE Transactions on Robotics

[3] Beni, G. And J. Wang. (1989). “Swarm intelligence”. At Seventh Annual Meeting of Swarm Intelligence.

[4] Michael Montemerlo.(2003)“A Factored Solution to the Simultaneous Localization and Mapping Problem"

[5] M. Montemerlo and S. Thrun. (2001)."An improved particle filtering algorithm for simultaneous localization and mapping that provably converges". In SODA '01: Proceedings of the twelfth annual ACMSIAM symposium on Discrete algorithms, pages 735-744

[6] M. Montemerlo, S. Thrun, D. Koller, and B. Wegbreit. Fastslam. (2002)."A factored solution to the simultaneous localization and mapping problem".

[7] S. Thrun, M. Montemerlo, D. Koller, B. Wegbreit, J. Nieto, and E. Nebot. (2004).”An efficient solution to the simultaneous localization and mapping problem with unknown data association".

[8] Maria Isabel Ribeiro.( 2004)." Kalman and Extended Kalman Filters Concept, Derivation and Properties" .Institute for Systems and Robotics Instituto Superior T'ecnicoAv. Rovisco Paris, 1 11049-001 Lisboa PORTUGAL

[9] Shoudong Huang.(2010).“Understanding Extended Kalman Filter”, ARC Centre of Excellence for Autonomous Systems (CAS), University of Technology Sydney

[10] Juan C. Muller, Yan Meng, Ọlọrundamilọla Kazeem. ( 2007). “A Hybrid ACO/PSO Control Algorithm for Distributed Swarm Robots". New Jersey City University, New Jersey, USA. Proceedings of the IEEE Swarm Intelligence Symposium SIS

[11] D. Payton, M. Daily, R. Estowski, M. Howard, and C. Lee. ( 2001)."Pheromone Robotics, Autonomous Robots". Vol. 11, No.3

[12] Yan Meng , Ọlọrundamilọla Kazeem, Juan C. Muller. ( 2007).”A Hybrid ACO/PSO Control Algorithm for Distributed Swarm Robots", Proceedings of the 2007 IEEE Swarm Intelligence Symposium . 